\title{
System management technology for technical maintenance of transport and technological machines
}

\author{
Vladimir Voronin* \\ Pacific State University, 680042, Khabarovsk, Russia
}

\begin{abstract}
The paper considers the generalized structure of maintenance system, including, in the general case, the protection subsystem, the functional diagnostics subsystem, the test diagnostics subsystem, the maintenance and repair subsystem. The technical implementation of the listed subsystems is possible in the form of tools built into the object and in the form of tools external to the object. For the generalized structure of the transport and technological machines maintenance system, the possible ratios of the volumetric characteristics of built-in and external subsystems are analyzed (using the simplest analytical expression). The possibility of formalizing the control process of a generalized system of technical maintenance of autonomous objects within the framework of a finiteautomaton model is discussed. In accordance with this model, it is proposed, first, to consider the on-board maintenance system as an operational automaton; second, each of its subsystems should be considered as a separate internal automaton that implements its own technological algorithm; third, the interaction of the four automata should be organized within the control automaton for the above-mentioned operating automaton. The algorithm for the functioning of this control automaton has been synthesized.
\end{abstract}

\section{Introduction}

The complex property "reliability" is one of the most important of the infinite set of properties for transport and technological machines. Complexity, first of all, is characterized by the fact that this property is essential for each stage of the life cycle. Such a cycle is implemented in a certain system, which is usually called a reliability assurance system and distinguishes a reliability design system in it, a production and technological system and a maintenance system $(M S)$.

The process of managing the reliability of a technical object $(T O)$, used for its intended purpose, can be implemented in $M S$ with different structure. A generalized version of the $M S$ structure is a four-circuit control system shown in Figure 1 [1].

\footnotetext{
*Corresponding author: 004183@pnu.edu.ru
} 


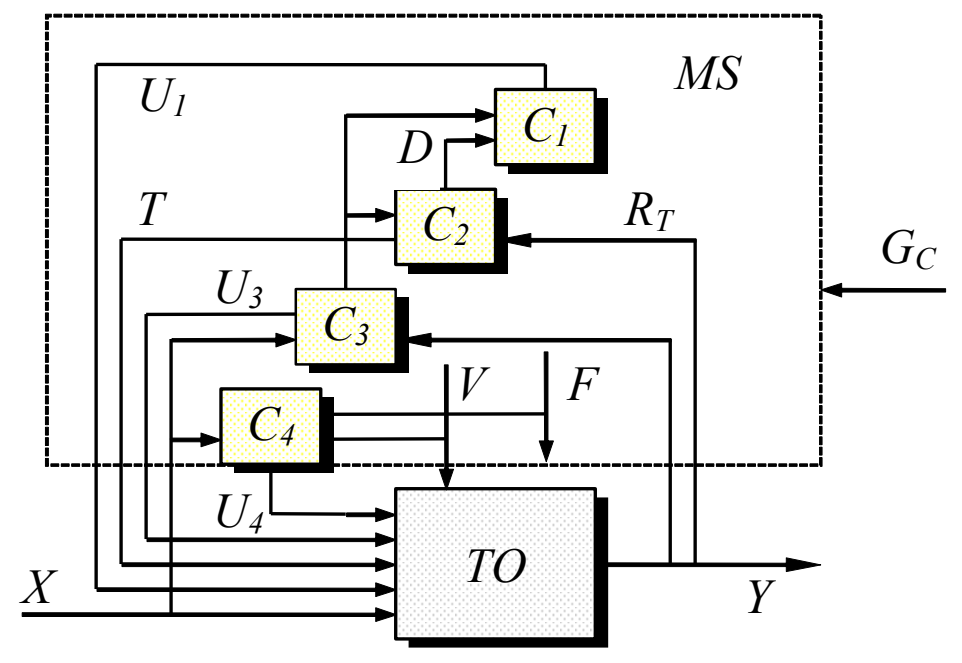

Fig. 1. Generalized $M S$ structure.

$M S$ traditionally includes four subsystems. Subsystem $C_{4}$ has the ability to assess the impact of the operating environment $V$, operational load of the facility $F$ and functional inputs $X$. When leaving $V, F, X$ out of range $C_{4}$ generates protective control action $U_{4}$, neutralizing effect of the latter. Such systems exist mainly as a part of an object and are called protection systems.

Subsystem $C_{3}$ based on the information analysis about the inputs $X$ and outputss $Y$ the object assesses the current technical condition $(T C)$ and when it goes beyond the permissible limits, forms a control action $U_{3}$ (activates backup capabilities) and / or transmits information about $T C$ deviation from the norm to subsystems $C_{l}$ or $C_{2}$. Such systems are called control systems or functional diagnostics systems and they, as a rule, are partially built into the object.

Subsystem $C_{2}$ has the ability to actively influence $T$ to an object through functional or other input channels, to perceive its reaction $R_{T}$ on this effect from the output functional channels or from special control points and from the analysis $T$ and $R_{T}$ assess the technical condition in the form of a diagnosis $D$. It is called a test diagnostics system and, in most cases, it exists separately from the object.

Subsystem $C_{l}$ based on $D$ and management objectives $G_{C}$ forms a control technical impact $U_{l}$ restorative nature. It is almost always external to the object, and it is customary to call it a maintenance and repair subsystem.

\section{Problem review and problem statement}

The generalized structure shown in Figure $1 M S$ typical for such transport and technological machines, within which a certain functional process of the "input-output" type (flow objects) is implemented. Objects of the opposite type (bridges, power transmission towers, buildings, etc.) have $M S$ significant structural features.

Technical implementation of systems $C_{1^{-}} C_{4}$ is possible in the form of technical means built into the object and in the form of technical means external to the object. The existing practice shows that in most cases a complex option is used [2] - part of the tools is built into 
the object, and the second part is used independently of it. This raises the problem of determining the values of volumetric indicators of the above-mentioned two MS parts (external and built-in), as well as the distribution of these values by the subsystems $C_{1}-C_{4}$.

The problem of the relationship between external and built-in infrastructure is typical for every sufficiently complex technical facility and its operating conditions. This problem is of particular relevance in relation to autonomous or partially autonomous objects, and it is widely discussed in the scientific and technical community. In confirmation of this fact, we give a brief overview for the relatively characteristic subject areas.

The work [3] considers the foundations of creating a new generation of technologies and systems for managing the life cycle of products for rocket-space and aviation technology based on the proactive control methodology. The conceptual basis of such control is to carry out proactive multivariate control of the technical state of an object based on information coming from built-in intelligent information sensors and devices.

The article [4] illustrates the results of a new approach development to the management of maintenance and repair processes for aerospace products. In this approach, each functional part of the object is associated with an agent, which is an autonomous program capable of responding to events, making decisions and interacting with other agents or a supersystem, without requiring external instructions, but implementing an internal built-in cycle for managing a technical state.

The authors of the publication [5] substantiate the need to create a multifunctional uninhabited underwater object with kinematic and cybernetic redundancy and propose to synthesize the control of this object, taking into account technological reliability and safety.

To solve a number of complex problems associated with the need to maintain, for example, the constant relative position of underwater vehicles in space in [6], it is recommended to use the method of "group management" control of several vehicles as a single control object. At the same time, the management system for such an object becomes significantly more complicated, and it is based on the principles of decentralized management.

The article [7] considers a new method of monitoring underground gas pipelines to prevent possible accidents on them due to gas leaks. It is proposed to use an autonomous gas analyzing station.

The work [8] summarizes the achievements of the known maintenance systems, analyzed a possible version of monitoring the life cycle of transport and technological mechatronic systems, based on the proposed algorithm for technical condition autonomous control.

The analyzed problem of a possible $M S$ structure is also relevant for other classes of technical objects [9].

The main tasks of this work are, first, the analysis of possible ratios of volumetric characteristics of $M S$ subsystems for standard and autonomous objects; second, the formalization of the control process of the generalized system of maintenance of autonomous objects within the finite-automaton model framework.

\section{Volumetric characteristics of MS subsystems}

For a formal analysis of the $M S$ various subsystems volumetric characteristics ratio, it is desirable to use some mathematical construction. In the simplest version, it might be possible to offer the function $W_{i}$, depending on the ratio of the conditional volumes of built-in and external means in the subsystem $C_{i}$, of the following simplest form

$$
W_{i}=V_{o i} /\left(V_{c i}+1\right)
$$


where $V_{o i}$ is the conditional amount of built-in funds, $V_{c i}$ is the conditional amount of external funds. Wherein $V_{o i}+V_{c i}=1$. Obviously, the values of this function belong to the segment within the boundaries 0 and 1 .

It is clear that for most of the typical transport and technological machines classes, the subsystems $C_{l}$ have the value $W_{l}$ in the surrounding area 0 , the subsystems $C_{4}$ have the value $W_{4}$ in the surrounding area 1 , and the subsystems $C_{2}$ and $C_{3}$ are located in the middle of the segment.

For special classes of objects (these include autonomous objects or objects with difficult operating conditions), the picture changes.

Let us consider a situation in which $W_{4}$ is less than one for typical objects as an example. Let the operating mode of the object include periodically storage sub-modes, then for such a case it makes sense to have external protection systems in relation to the object, neutralizing the negative influence of the environment. This decreases the value $W_{4}$.

An example of a symmetrical situation for $C_{l}$ a typical object is that $W_{l}$ has a value greater than zero. Let the object be a recoverable object, then its internal structure can include the elements increasing the maintainability level, which can be logically attributed to the builtin part of the subsystem $C_{1}$. For the subsystem $C_{2}$ a typical built-in part is the means to ensure the control points organization.

In the considered ratio of internal and external means, autonomous objects have significant distinctive features in terms of automating the subsystems $C_{1}-C_{4}$ functions.

In the subsystems $C_{2}, C_{3}$ and $C_{4}$ the automation of all the necessary functions is fundamentally straightforward, i.e., there is a real opportunity to provide the technical and technological values of the elements $\left\{W_{2}, W_{3}, W_{4}\right\}$ close to one.

In relation to the subsystem $C_{l}$ the task is more complicated, but it is currently quite successfully solved by using, first of all, various types of redundancy (reservation of resourcelimiting blocks) and special compensation devices [10].

Thus, for autonomous technical objects, in which the values of the elements of the set $\left\{W_{1}, W_{2}, W_{3}, W_{4}\right\}$ provided close enough to one, there is a problem of formalizing the relationship between subsystems $C_{1}-C_{4}$. In other words, it is necessary to organize formal effective management of the built-in maintenance system (to ensure the automatic interaction of all $M S$ subsystems). A possible option for such control is proposed in the next section.

Lots of $\left\{W_{1}, W_{2}, W_{3}, W_{4}\right\}$ actually characterizes the distribution of the reliability load between the reliability design system and the production and technological system, on the one hand, and $M S$, from the other hand. It is appropriate here to formulate the following nontrivial question. On what basis are the decisions on such distribution made? In the monograph [1], a list of the main indicators of the technical task is formed, which makes it possible to make a decision on dividing the reliability of an object into two types "hereditary reliability" and "external additional reliability".

\section{Formalization of the maintenance system management process}

Maintenance systems for autonomous objects are almost completely built into these objects. It is obvious that the control of the subsystems $C_{1}-C_{4}$ interaction should be implemented automatically. The implementation of automatic control systems is most often based on the theory of finite automata [11].

Among the varieties of finite-automata models for practical implementations of control systems, a two-element model is most often chosen, including a control automaton and an operational automaton.

In accordance with the two-element model, it is proposed, first, an onboard $M S$ considered as an operational automaton; second, each of the subsystems $C_{1}-C_{4}$ should be considered as 
a separate automaton that implements its own technological algorithm; third, the interaction of internal automata $C_{1}-C_{4}$ is organized within the control machine for the above-mentioned operating machine.

Thus, the automaton model is used in two versions, namely: external and universal - in general $M S$ is considered as an operational automaton and requires a control automaton as well as an internal one, specialized for each of the subsystems $C_{1}-C_{4}$, which specialization is determined by the particular autonomous object characteristics.

Graphical illustration of the proposed in this work finite-automaton approach for $M S$ autonomous objects control is shown in Figure 2.

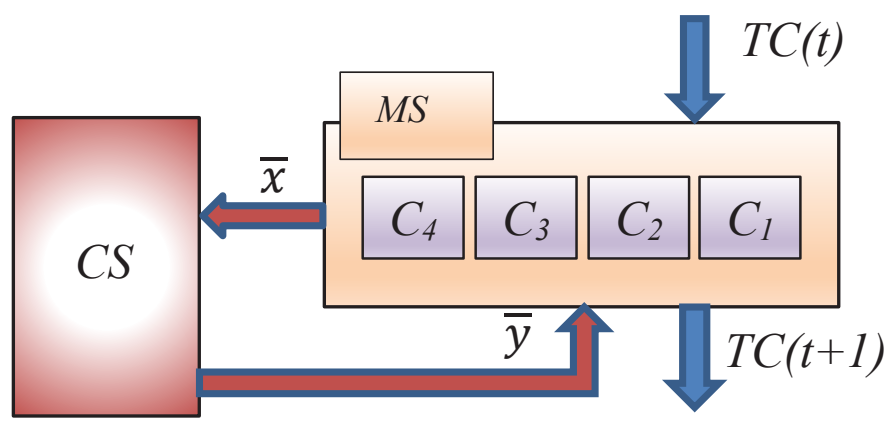

Fig. 2. $M S$ automatic onboard model.

Control machine (Figure $2 C S$ - control system) provides interaction of subsystems $C_{l^{-}} C_{4}$ by the vector $\bar{y}$, moreover, the components of the vector $\bar{y}$ are generated taking into account the state vector $\bar{x}$, which consists of four independent zones $V_{i}$ - one for each subsystem $C_{l}-C_{4}$. The system operates in discrete time, the cycle time of which is determined as follows.

Positive result of polling the state of the subsystem $C_{4}$ (the control or protection subsystem has been triggered) starts a cycle for handling an abnormal situation. The result of the cycle is the transition of the technical state of the object from $T C(t)$ to $T C(t+1)$. The average cycle time limits the clock parameters. The cycle time must exceed the sync pulse duration by at least 4 times.

The following is an algorithm for handling an abnormal situation. The algorithm includes the possibility of accessing the supersystem (data request $V_{0}$ ) in the event of a dead-end.

Step 1. Transfer control to the subsystem $C_{4}$.

Step 2. State vector analysis $V_{4}$ of the subsystem $C_{4}$. If the conjunction of the elements $V_{4}$ is equal to True, then to Step 2, otherwise to Step 3 (Unnominal situation).

Step 3. Transfer control to the subsystem $C_{3}$. If the conjunction of the elements $V_{3}$ is equal to True, then to Step 4, otherwise to Step 5.

Step 4. Data request $V_{0}$ from the supersystem. If $V_{0}$ is equal to True, then the scope of operation ability monitoring should be expanded to Step 3, otherwise to Step 10.

Step 5. Transfer control to the subsystem $C_{2}$. If the conjunction of the elements $V_{2}$ is equal to True, then to Step 6, otherwise to Step 7.

Step 6. Data request $V_{0}$ from the supersystem. If $\mathrm{V}_{0}$ is equal to True, then the set of possible defects should be expanded to Step 5 otherwise to Step 10.

Step 7. Transfer control to the subsystem $C_{1}$. If for all $V_{2}$ zero elements there are spare replacement elements, then to Step 9, otherwise to Step 8.

Step 8. Data request $V_{0}$ from the supersystem. If $V_{0}$ is equal to True, then to Step 9, otherwise, block sync pulses and to Step 10.

Step 9. Turn on spare replacement elements and to Step 10.

Step 10. Unnominal situation has been handled. Change-over to Step 1. 
According to the algorithm proposed above, in accordance with the technology of synthesis of control automata, a graph-scheme was constructed, the labeling of which determined six internal states of the Mealy machine. Then, in accordance with the technology of the canonical method of structural synthesis, an automaton table and a logical diagram of the $M S$ control system have been built.

\section{Conclusion}

$M S$ building technology control system satisfies the system-wide principle of downstream design, namely: the external level of the system uses universal technology, and the internal level uses specialized automata. The proposed system can be extended to automated and manual construction options. In the subsystem $C_{2}$ it is recommended to use the algorithms for finding the defects from [12-13].

\section{References}

1. V.V. Voronin, Theoretical problems of diagnostic expert systems (Dal'nauka, Vladivostok, 2005).

2. M.Yu. Okhtilev, B.V. Sokolov, R.M. Yusupov, Intelligent technologies for monitoring and controlling the structural dynamics of complex objects. Series: Computer Science: Unlimited Possibilities and Possible Limitations (Science, M., 2006).

3. M.Yu. Okhtilev, B.V. Sokolov, R.M.Yusupov, M.M. Styskin, Zhao V.Yu.-D., Bulletin of the Universities Instrument Engineering 63(2), 187-190 (2020).

4. O.I. Lakhin, E.V. Simonova, P.O. Skobelev, A.S. Polnikov, Yu.S. Yurygina, Information and control systems 4(77), 10-16 (2015).

5. M.N. Plavinskiy, A.S. Protsenyuk, E.Yu. Smirnova, S.A. Polovko, S.S. Kavtrev, Investigation of the ways to create hyper-redundant unmanned underwater vehicles, In the collection: Materials of the XII Multiconference on Management Problems (MCMP2019). Taganrog: Publishing House of the Southern Federal University 2, 161-163 (2019).

6. D.O. Semenov, I.A. Mokhov, B.A. Luskin, Sudostroenie 4(845), 22-24 (2019).

7. A.V. Shalygin, E.V. Akhmerov, E.D. Karyakina, Mining information and analytical bulletin (scientific and technical journal) S5-2, 449-455 (2017).

8. A.V. Kudrevatykh, N.V. Kudrevatykh, Diagnostics of the actual technical condition of the gearboxes of excavator-automobile complexes (Publishing house of the Kuzbass state. tech. un., Kemerovo, 2018).

9. M.V. Asalkhanov, V.P. Kolinichenko, O.V. Yakushenko, D.V. Sinkin, National Association of Scientists 3-1 (19), 10-12 (2016).

10. A.K. Tugengol'd, R.N. Voloshin, A.R. Yusupov, T.N. Kruglova, Bulletin of the Don State Technical University 19(1), 74-80 (2019).

11. V.M. Glushkov, Synthesis of digital automata (GIFML, M., 1962).

12. V.D. Bloshchinskiy, S.V. Shalobanov, S.S. Shalobanov, Application Of Configurable Diagnostic Models On IIR-filters And Laguerre Filters For Finding Parametric Defects, In Continuous Dynamic Objects, 2019 International Siberian Conference on Control and Communications (SIBCON), 1 - 5 (2019). 
13. S.V. Shalobanov, S.S. Shalobanov, Diagnostics of automatic control systems using trial deviations of model parameters and binary diagnostic signs, 2020 International Russian Automation Conference (RusAutoCon), 1-5 (2020). 